\title{
Introduction : l'économie de la connaissance
}

\author{
Lise Verlaet* \& Vincent Liquète**
}

* Université de Montpellier 3, LERASS-Céric (EA 827)

** Université de Bordeaux, IMS- Equipe Représentations, Usages, Développements \& Ingénieries de l'Information (RUDII), UMR 5218 - CNRS

Avec ce deuxième volet de la thématique «De la gestion de la connaissance à l'économie de la connaissance », l'accent sera mis sur l'économie de la connaissance. Comme nous avons pu le constater à travers le premier numéro consacré à la gestion des connaissances ${ }^{1}$, l'économie des connaissances - ou de l'immatériel - est étroitement liée aux technologies de l'information et de la communication, à la sphère numérique et plus spécifiquement à l'Internet; corroborant par là même les dires de Benghozi, Bergadaà, et Gueroui ${ }^{2}$. Cette économie de l'immatériel repose sur un bien difficilement quantifiable : les connaissances. Selon Delamotte ${ }^{3}$, « la nature du savoir et sa circulation pose de nombreux problèmes de définition, notamment aux économistes et aux sociologues ». Les chercheurs en sciences de l'information et de la communication, comme ceux des sciences de gestion, n'échappent pas à cette difficulté de définir mais également de quantifier l'économie de la connaissance. D'une part, car la connaissance ne peut être considérée comme une denrée en particulier parce que celle-ci est le produit d'une construction intellectuelle inachevée et permanente, en perpétuelle modification et hybridation des contenus. D'autre part, parce que les connaissances (au sens de biens d'apprentissage) ne sont pas commensurables puisqu'elles « ne produisent pas sur les individus des résultats homogènes »; il devient dès lors très difficile de considérer les enrichissements de la connaissance tant sur un plan cognitif, qu'économique ou informationnel. Nonaka et Takeuchi ${ }^{4}$ distinguent les connaissances explicites des connaissances tacites, les unes sont objectives et s'expriment aisément de manière formelle, les autres sont plus subjectives, difficiles à formuler et intrinsèquement liées à l'expérience des individus. Or comme le soulignent ces auteurs, ce sont tout particulièrement les connaissances tacites qui sont à fortes valeurs ajoutées et fondamentales pour l'innovation et la compétitivité. Bien que datant, cette approche fondamentale de la gestion de connaissances reste d'actualité. En effet, comment capter ces connaissances tacites ? Comment circulent et se transmettent-elles? Nous verrons à travers les articles de ce numéro qu'au-delà des dispositifs socio-techniques numériques, qui ne sont in fine que des outils au service de l'homme ${ }^{5}$, la véritable clé de voûte de la production, de la diffusion, du partage et de la valorisation des connaissances humaines repose sur l'interaction entre les acteurs et le sens de ces connaissances dans des contextes ou démarches collectives du rapport aux savoirs. À cet égard, l'économie des connaissances est nécessairement

\footnotetext{
${ }^{1}$ Mallowan, M., Liquète, V., Verlaet, L., (2015) Gestion des connaissances. Revue Communication \& Management, Vol.12 (1/2015).

${ }^{2}$ Benghozi, P. J., Bergadaà, M., \& Gueroui, F. (2014). Les temporalités du web. De Boeck Superieur.

${ }^{3}$ Delamotte, E. (2004). «Introduction : de l'économie de la connaissance à la société de la connaissance ». In $D u$ partage au marché : Regards croisés sur la circulation des savoirs, sous la direction d'Eric Delamotte, Presses Universitaires du Septentrion, p.32.

${ }^{4}$ Nonaka, I., \& Takeuchi, H. (1995). The knowledge-creating company: How Japanese companies create the dynamics of innovation. Oxford university press.

${ }^{5}$ Gallot, S. \& Verlaet, L. (2016). La transparence : l'utopie du numérique. Revue Communication et Organisation, n $49 / 2016$, p.203-217.
} 
à considérer selon une perspective informationnelle, communicationnelle et cognitive.

Nous commencerons ce numéro avec les travaux de Marc Tanti qui présentent la dimension économie de la connaissance du système de gestion des connaissances développé par les armées françaises pour manager l'information sur le risque sanitaire pouvant toucher les forces sur les terrains d'opération. Cet article fait état d'un retour d'expérience sur le développement et l'alimentation d'un système de gestion des connaissances pour les armées, et notamment, de la nécessaire organisation horizontale entre les acteurs d'un tel type de projet. Chaque acteur participant au développement du dispositif représente un corps de métier et assure dans cette coconception du système d'information la fonction de médiateur: des documents pour le cyberdocumentaliste, des contenus pour les experts, des usages pour l'ergonome. Ces connaissances individuelles, mais néanmoins interreliées, sont assemblées par les informaticiens pour créer un «objet unique de travail collectif». Si ce système de gestion des connaissances a pour vocation première d'aider les décideurs dans leur prise de décision, il s'avère qu'à l'usage la capitalisation des connaissances permet de diminuer les coûts, de gagner en performance et en création de valeur, et assure à l'usager une meilleure gestion de son propre savoir. Nous pourrons noter que dès lors qu'il s'agit de production de connaissances, et aussi rigide et descendante soit la structure de commandement initiale de l'organisation, celle-ci cède le pas à une structure plus souple, plus collaborative. Les connaissances ne s'imposent pas, elles se co-construisent.

Nous poursuivrons avec les recherches de Maryline Margueritte qui considère à travers une approche systémique La place du Knowledge Management dans les organisations de santé. L'auteur marque une corrélation forte entre culture organisationnelle et Knowledge Management, et interroge subséquemment la notion de communauté (de pratique, d'apprentissage, scientifique, virtuelle, « on line »), laquelle donne un sens à la construction informationnelle collective. Cette recherche soulève qu'en réponse aux bouleversements technologiques imposés par les organisations de santé, les acteurs ont tissé des liens sociaux qui les ont peu à peu conduits à constituer différentes formes de communautés notamment pour pallier les dysfonctionnements perçus du système dont pâtissaient les patients. Après avoir démontré l'importance de la récursivité des relations entre acteurs dans les organisations de santé, cet article souligne que la mutualisation des connaissances est indispensable, d'une part pour éviter la dispersion des compétences et d'autre part pour assurer la performance à la fois au niveau individuel mais également collectif et consécutivement de l'organisation. Les besoins des acteurs de santé n'étant pas tant sur la préservation des connaissances mais davantage axés sur le partage et surtout la coordination de connaissances, la logique inhérente au Knowledge Management reposant uniquement sur la capitalisation de connaissances ne répond pas complètement à leurs pratiques de gestion en réseaux. Par ailleurs, l'auteur insiste sur la notion confiance, posée comme déterminante pour que le partage de connaissances puisse se faire, et fait la part belle à l'apprentissage interactionnel, lequel vient renforcer la coordination et réciproquement. Et si, de prime abord, cet apprentissage interactionnel semble se faire au détriment de l'apprentissage organisationnel, il n'en est rien. Car c'est le collectif humain (et sa coordination) qui vient nourrir et renouveler l'organisation, qui garantit sa performativité.

Après avoir exploré la gestion et l'économie des connaissances dans le secteur des armées et de la santé, nous focaliserons notre attention sur le secteur de l'enseignement et de la recherche, avec en premier lieu les travaux d'Hans Dillaerts qui nous amèneront à nous questionner sur l'Ouverture et partage des résultats de la recherche dans l'économie de la connaissance 
européenne: quelle(s) liberté(s) de circulation pour l'IST. Ces recherches mettent en évidence les difficultés liées au libre accès et aux modèles économiques inhérents, lesquelles viennent exacerber les convictions des différentes parties prenantes. Les uns voyant dans le libre accès la promesse d'une science ouverte, collaborative voire citoyenne où la connaissance scientifique serait un bien commun. Quand les seconds se réjouissent à l'idée de pouvoir investir dans des domaines jusque-là protégés notamment par les brevets. Comme le démontre cet article, face à cette dichotomie les politiques européennes ne savent ou ne veulent prendre position, confrontant ainsi les chercheurs à des injonctions paradoxales. En effet, d'une part, les politiques européennes prônent le libre accès aux publications et données de la recherche, mettent en avant la science ouverte et subséquemment l'innovation ouverte, laissant à penser que les résultats de la recherche scientifique doivent être considérés comme des biens communs. Et d'autre part, ces mêmes politiques enjoignent les chercheurs dans le cadre de l'évaluation de leurs travaux à publier dans des revues à facteur d'impact - lesquelles sont pour la plupart entre les mains des éditeurs commerciaux - ainsi qu'à déposer des brevets pour valoriser les résultats de leur recherche. Les notions de " communs scientifiques », d' " inter ou transdisciplinarité », de " science ouverte et citoyenne », etc. peuvent-elle dès lors avoir un sens tant que les politiques d'évaluation de la recherche restent sur les critères d'un système aux valeurs antinomiques?

Nous poursuivrons dans le secteur de l'enseignement et la recherche avec Le chercheur en humanités digitales : un cas particulier de travailleur du savoir, étude réalisée par Olivier Le Deuff sur l'évolution des méthodes et des stratégies de gestion des chercheurs en humanités numérique. L'auteur pose le chercheur comme un travailleur du savoir des humanités digitales, soit un travailleur qui utilise ses facultés intellectuelles pour créer de l'information et des connaissances dans ses activités quotidiennes et qui recourt aux technologies informatiques ou numériques pour l'accompagner dans les différentes facettes de cette production (recherche et traitement des informations, communication). À ce titre, le chercheur en humanités numériques renouvelle les formes d'écriture et de productions scientifiques notamment la production, le traitement et la diffusion de données, lesquelles ne sont que peu voire pas valorisées, ni évaluées par les institutions. Or l'économie des connaissances des humanités digitales nécessite d'ouvrir les logiques de publication traditionnelle aux modèles de productions issus du numérique. Cette mutation des logiques de production est également prégnante dans les profils des chercheurs en humanités numériques, davantage tournés vers des pratiques inter voire transdisciplinaire, vers des savoirs certes théoriques mais également techniques, et pour lesquelles la polyvalence et la collaboration sont les maîtres-mots. Ceci influe également sur les modes de travail et d'accès à la connaissance. Le risque pointé par l'auteur est de se laisser submerger par la loi du cool et tous les phantasmes liés aux capacités du numérique dans l'exploitation des données. Quoi qu'il en soit, l'étude d'Olivier Le Deuff souligne que le chercheur en humanités digitales mobilise de nouveaux modes de travail pour l'organisation des connaissances laissant par là même entrevoir un changement épistémologique.

Toujours dans le secteur de la recherche, Marie-Claude Bouhedi nous fera part de l'étude qu'elle a conduite au sein de l'INRA sur Les pratiques de partages des connaissances d'une unité de recherche pluridisciplinaire en interne et externe vient confirmer le fait que la dissémination de la recherche scientifique et des données se heurte aux logiques de confidentialité. L'auteur, qui s'intéresse aux outils et aux habitus technologiques des chercheurs, a pu observer une certaine réticence aux changements technologiques et plus largement aux changements de modalités scientifiques, lesquels sont perçus par les acteurs comme une perte d'avantages concurrentiels. 
Dans la même veine, l'enquête et les observations réalisées auprès des chercheurs montrent qu'il n'y a partage de connaissances que lorsque les acteurs sont en situation de confiance réciproque, que les problématiques liées à la propriété intellectuelle, à la primauté des idées et à la brevetabilité des recherches entrainent une compétition chez les acteurs, génère un climat de méfiance et de suspicion qui in fine peut se révéler néfaste pour l'innovation, la recherche et le développement. De plus, la publication scientifique est l'élément indispensable pour le chercheur dans sa quête de légitimité : un article entraîne la reconnaissance par les pairs, génératrice de collaborations potentielles et d'éventuelles subventions. Comme le souligne Bourdieu', celui qui publie se retrouve donc dans une compétition permanente pour être reconnu scientifiquement et socialement par ses pairs. La solution serait-elle de concevoir des systèmes de gestion des connaissances, qui outre les aspects liés à la capitalisation des connaissances, assureraient la traçabilité de la propriété intellectuelle, genre de workflow de la connaissance scientifique, des artefacts scientifiques?

Nous terminerons ce numéro avec les travaux de Thibaud Hulin qui propose un modèle pour la formation aux activités numériques. Il défend l'idée que le modèle par la gestion procédurale des connaissances n'est plus suffisant et propose de le compléter par le management réflexif, ce dernier est appliqué dans le cadre d'un dispositif de formation professionnelle aux usages du numérique. Par extension, ce changement de modèle revient, selon l'auteur, à ne plus seulement considérer le how-to (comment faire) mais y adjoindre le how-know (savoir-faire) plus enclin à mobiliser le sens critique et l'autonomie des acteurs. Pour Thibaud Hulin, « le management des connaissances numériques suppose de développement d'une culture numérique comme conscience de soi en situation de médiation numérique ». Dans la perspective de mieux former l'acteur à ses activités numériques, l'auteur propose un modèle sous forme de couches lesquelles représentent les différentes interactions numériques humaines.

Comme vous pourrez le constater, les six articles retenus démontrent également que la production de biens immatériels induit des changements sur la structure des organisations et leurs modes de gouvernance. Ces dernières ne peuvent résolument plus être appréhendées de manière pyramidale mais a minima horizontale si ce n'est selon des modalités réticulaires et/ou virtuelles. Cette restructuration n'est pas sans incidence sur la culture des entreprises et leur système de management. Subséquemment, ces recherches soulignent que l'appropriation et les changements s'opèrent dans un premier temps au niveau individuel avant d'impacter les niveaux organisationnels et institutionnels. Les cartes sont donc entre les mains des chercheurs, des managers et plus largement des acteurs de la connaissance au sein des diverses natures d'organisations.

\footnotetext{
${ }^{6}$ Bourdieu, P. \& Waquant, L.D.J. (1992), Réponses, pour une anthropologie réflexive, Editions du Seuil, Paris.
} 